\title{
Evaluation of Biosimilarity Based on an Empirical Bayes Method
}

\section{Hsiao-Hui Tsou ${ }^{1,2 *}$, Chi-Tian Chen ${ }^{3}$, Chin-Fu Hsiao ${ }^{1,4}$ and Yu-Chieh Cheng ${ }^{1}$}

${ }^{1}$ Division of Biostatistics and Bioinformatics, Institute of Population Health Sciences, National Health Research Institutes, Miaoli, Taiwan

${ }^{2}$ Graduate Institute of Biostatistics, College of Public Health, China Medical University, Taichung, Taiwan

${ }^{3}$ Technical Operation, StatPlus, Inc., Taipei, Taiwan

${ }^{4}$ Division of Clinical Trial Statistics, Institute of Population Health Sciences, National Health Research Institutes, Miaoli, Taiwan

\begin{abstract}
Biosimilars have received much attention from sponsors and regulatory authorities while patents on many biological products had expired recently or will soon expire in the next few years. According to the definition of biosimilar product from the European Medicines Agency's guidance and the U.S. Food and Drug Administration's guidelines, biosimilar should be highly similar, not identical, to the innovative biological product. In this research, we focus on establishing posterior criterion to assess the biosimilarity between the biosimilar product and the innovator product. We consider the prior information of the reference product and a non-informative prior to build the mixture empirical prior information of the biosimilar product. We further construct a posterior criterion to check the biosimilarity between the reference product and the biosimilar product. If the posterior probability of the similarity criterion is higher or equal to a pre-specified level, the biosimilarity between the reference product and the biosimilar product will be concluded. The statistical properties of the proposed approach are discussed through numerical results in different scenarios. A real example is provided to illustrate applications of the proposed approach.
\end{abstract}

Keywords: Biosimilar; Biosimilarity; Mixture prior; Empirical bayes; Posterior criterion

\section{Introduction}

Biosimilars have attracted much attention from sponsors and regulatory authorities while patents on many biological products had expired recently or will soon expire in the next few years. According to the report of Evaluate Pharma World Preview 2017, Outlook to 2022, the proportion of drug sales that have been listed in the world's top 100 pharmaceutical companies has continued to increase, reaching $26 \%$ in 2016 and $30 \%$ in 2022 [1]. Many patents of the world's best-selling biological products have expired since 2015 or are going to expire in recent years. According to a new market research report published by MarketsandMarkets, the global biosimilars market is expected to reach USD 10.90 Billion by 2021 from USD 3.39 Billion in 2016, at a CAGR of $26.3 \%$ during the forecast period [2].

Unlike the chemically synthesized drugs, biological drugs are much more complicated with larger size and complicated structure. Biological drugs can be sensitive to environmental conditions such as temperature or pressure, and may expose patients to immunogen reactions. The European Medicines Agency (EMA) of the European Union (EU) has published a guideline on similar biological medicinal products for approval of these products since 2005 [3]. On February 9, 2012, the US Food and Drug Administration (FDA) issued three draft guidance documents on biosimilar product development to assist industry in developing such products in the United States [4]. According to the definition of biosimilar product in these guidelines, the biosimilar should be highly similar, but not identical, to the innovative biological product. In these guidelines, however, no specific statistical methods for assessment of biosimilarity in clinical trials were mentioned.

Some literatures published in recent years to deal with the development of statistical methodology for evaluation of "biosimilarity" between biosimilar products and innovator's biologics [5-14]. As indicated by Chow et al. [7], current regulation for assessment of bioequivalence may be too loose to be applied for assessment of biosimilarity. Other statistical methodologies for evaluation of biosimilarity from different approaches are recommended.
Hsiao et al. [15] proposed a Bayesian approach for assessing the similarity of bridging studies. In this paper, we develop an empirical Bayes approach for statistical evaluation of similarity between a biosimilar product and the innovator biologic using the similar idea from Hsiao et al. [15]. We established a similarity criterion and derived the posterior distributions of treatment effect of the innovator biological product and the biosimilar, respectively. The statistical properties of the proposed approach are discussed through numerical results in different scenarios. We provided a real example to illustrate applications of the proposed approach.

\section{Empirical Bayes Method}

Let $R_{i}$ and $B_{j}$ be the efficacy responses for the $i^{\text {th }}$ subject and $j^{\text {th }}$ subject receiving the innovator biological product and the biosimilar, respectively, $i=1, \ldots, N_{R}, j=1, \ldots, N_{B}$. Assume that $R_{i} \sim N\left(\mu_{R}, \sigma_{R}^{2}\right)$ and $\mathrm{B}_{\mathrm{i}} \sim \mathrm{N}\left(\mu_{\mathrm{B}}, \sigma_{\mathrm{B}}^{2}\right)$, where $\mathrm{N}\left(\mu, \xi^{2}\right)$ represents a normal distribution with mean $\mu$ and variance $\xi^{2}$. We assume that the unobservable real valued efficacy response $\mu_{\mathrm{R}}$ of the innovator biological product have a prior distribution of $N\left(\theta, \tau^{2}\right)$, that is, $\mu_{R} \sim N\left(\theta, \tau^{2}\right)$. On the other hand, for the prior information of $\mu_{\mathrm{B}}$, we consider a mixture model which is a weighted average of two priors as given below:

$$
\pi\left(\mu_{B}\right)=\gamma \pi_{1}\left(\mu_{B}\right)+(1-\gamma) \pi_{2}\left(\mu_{B}\right)
$$

where $0 \leq \gamma \leq 1$. In above mixture prior, $\pi_{2}($.) is a normal prior with

*Corresponding author: Hsiao-Hui Tsou, Division of Biostatistics and Bioinformatics, Institute of Population Health Sciences, National Health Research Institutes, 35 Keyan Road, Zhunan, Miaoli County 350, Taiwan, Fax: 886-37-586-467, Tel: 886-37-246-166 Ext: 36181; E-mail: tsouhh@nhri.org.tw

Received November 15, 2017; Accepted December 17, 2017; Published December 27,2017

Citation: Tsou HH, Chen CT, Hsiao CF, Cheng YC (2018) Evaluation of Biosimilarity Based on an Empirical Bayes Method. J Bioequiv Availab 10: 7-10. doi: 10.4172/ jbb.1000367

Copyright: (c) $2018 \mathrm{Tsou} \mathrm{HH}$, et al. This is an open-access article distributed unde the terms of the Creative Commons Attribution License, which permits unrestricted use, distribution, and reproduction in any medium, provided the original author and source are credited. 
mean $\theta$ and variance $\tau^{2}$, whereas $\pi_{1}($.$) is a normal prior with mean 0$ and variance $\tau^{2}$.

A $\gamma$ value of 0 in the proposed mixture model in Eqn (1) indicates that the prior $\pi$ is equivalent to the prior used in the innovator biological product, while $\gamma$ being 1 indicates that no strength of the evidence for the efficacy provided by the innovator biological product.

Let $\hat{\mu}_{R}=\sum_{i=1}^{n_{R}} R_{i} / n_{R}$ be an estimator of efficacy response $\mu_{\mathrm{R}}$ of the innovator biological product. Similarly, let $\hat{\mu}_{B}=\sum_{j=1}^{n_{B}} B_{j} / n_{B}$ be an estimator of efficacy response $\mu_{\mathrm{B}}$ of the biosimilar. It follows that $\hat{\mu}_{R} \mid \mu_{R} \sim N\left(\mu_{R}, \sigma_{R}^{2} / n_{R}\right)$ and $\hat{\mu}_{B} \mid \mu_{B} \sim N\left(\mu_{B}, \sigma_{B}^{2} / n_{B}\right)$. Hence, the marginal sampling density of $\hat{\mu}_{R}$ is $N\left(\theta, \tau^{2}+\sigma_{R}^{2} / n_{R}\right)$. Given the data of the innovator biological product and prior information, the posterior distribution of $\mu_{\mathrm{R}}$ is Eqn (2):

$$
\pi\left(\mu_{R} \mid \hat{\mu}_{R}\right)=\frac{1}{\sqrt{2 \pi \frac{\tau^{2} \sigma_{R}^{2} / n_{R}}{\tau^{2}+\sigma_{R}^{2} / n_{R}}}} \exp \left[\frac{\left(\mu_{R}-\frac{\hat{\mu}_{R} \tau^{2}+\theta \sigma_{R}^{2} / n_{R}}{\tau^{2}+\sigma_{R}^{2} / n_{R}}\right)^{2}}{2 \frac{\tau^{2} \sigma_{R}^{2} / n_{R}}{\tau^{2}+\sigma_{R}^{2} / n_{R}}}\right]
$$

For the choice of above mixture prior, the marginal density of $\mu_{\mathrm{B}}$ is $N\left(\theta, \tau^{2}+\sigma_{B}^{2} / n_{B}\right)$. Given the data of the biosimilar and mixture prior information, the posterior distribution of $\mu_{\mathrm{B}}$ in Eqn (3):

$$
\begin{aligned}
\pi\left(\mu_{B} \mid \hat{\mu}_{B}\right) & =\gamma \frac{1}{\sqrt{2 \pi \frac{\tau^{2} \sigma_{B}^{2} / n_{B}}{\tau^{2}+\sigma_{B}^{2} / n_{B}}}} \exp \left[\frac{\left(\mu_{B}-\frac{\tau^{2} \hat{\mu}_{B}}{\tau^{2}+\sigma_{B}^{2} / n_{B}}\right)^{2}}{2 \frac{\tau^{2} \sigma_{B}^{2} / n_{B}}{\tau^{2}+\sigma_{B}^{2} / n_{B}}}\right]+ \\
+(1-\gamma) \frac{1}{\sqrt{2 \pi \frac{\tau^{2} \sigma_{B}^{2} / n_{B}}{\tau^{2}+\sigma_{B}^{2} / n_{B}}}} & \exp \left[\frac{\left(\mu_{B}-\frac{\tau^{2} \hat{\mu}_{B}+\theta \sigma_{B}^{2} / n_{B}}{\tau^{2}+\sigma_{B}^{2} / n_{B}}\right)^{2}}{2 \frac{\tau^{2} \sigma_{B}^{2} / n_{B}}{\tau^{2}+\sigma_{B}^{2} / n_{B}}}\right]
\end{aligned}
$$

The joint posterior distribution of $\left(\mu_{R}, \mu_{B}\right)$ would be in Eqn (4):

$$
\pi\left(\mu_{B}, \mu_{R} \mid \hat{\mu}_{B}, \hat{\mu}_{R}\right)=\pi\left(\mu_{B} \mid \hat{\mu}_{B}\right) \times \pi\left(\mu_{R}, \hat{\mu}_{R}\right)
$$

\section{Similarity Criterion}

Similarity on efficacy for the biosimilar can be concluded if the following posterior probability $\mathrm{P}_{\mathrm{sp}}$ is larger than a pre-specified limit $\lambda$, say $80 \%$ or $90 \%$, Eqn (5):

$$
P_{s p}=\operatorname{Pr}\left(\rho \mu_{R}<\mu_{B}<\mu_{R} / \rho \mid \text { data }\right)>\lambda
$$

where $\rho$ is defined as a limit for allowing the similarity.

In order to obtain the MLEs of $\left(\theta, \tau^{2}\right)$, we consider a historical information of the innovator drug, say $R_{H}$. When $\gamma=0$, the joint marginal sampling distribution of $\hat{\mu}_{R}$ and $\hat{\mu}_{R_{H}}$ is Eqn (6):

$$
m\left(\hat{\mu}_{R_{H}}, \hat{\mu}_{R}\right)=N\left(\theta, \tau^{2}+\frac{\sigma_{R_{H}}^{2}}{n_{R_{H}}}\right) N\left(\theta, \tau^{2}+\frac{\sigma_{R}^{2}}{n_{R}}\right)
$$

The logarithmic marginal sampling density is

$$
\begin{aligned}
& l_{\theta, \tau^{2}}\left(\hat{\mu}_{R}, \hat{\mu}_{B}\right)=-\ln (2 \pi)-\frac{1}{2} \ln \left(\tau^{2}+\frac{\sigma_{B}^{2}}{n_{B}}\right)-\frac{1}{2} \ln \left(\tau^{2}+\frac{\sigma_{R}^{2}}{n_{R}}\right) \\
& -\frac{\left(\mu_{R}-\theta\right)^{2}}{2\left(\tau^{2}+\frac{\sigma_{R}^{2}}{n_{R}}\right)}-\frac{\left(\mu_{B}-\theta\right)^{2}}{2\left(\tau^{2}+\frac{\sigma_{B}^{2}}{n_{B}}\right)}
\end{aligned}
$$

The maximum likelihood estimates of $\left(\theta, \tau^{2}\right)$ are the simultaneous solutions of the

following two equations:

$$
\begin{aligned}
& \frac{\partial}{\partial \theta} l_{\theta, \tau^{2}}\left(\hat{\mu}_{R_{H}}, \hat{\mu}_{R}\right)=0 \\
& \frac{\partial}{\partial \tau^{2}} l_{\theta, \tau^{2}}\left(\hat{\mu}_{R_{H}}, \hat{\mu}_{R}\right)=0
\end{aligned}
$$

We use Newton's method to determine the MLE. By a familiar empirical Bayes method, we replace $\theta$ and $\tau^{2}$ with the MLEs.

\section{Numerical Results}

The following numerical results are used to illustrate applications of the proposed method and discover the pattern between the $\mathrm{P}_{\mathrm{sp}}$ and the parameters corresponding to the mixture prior and the similarity criterion. First, we need to find the MLEs of the parameters $\left(\theta, \tau^{2}\right)$ of the normal prior. According to the result of the trial and a historical result of the reference product, the MLEs can be obtained by Eqn. (7) and (8). Here, we assume that $\hat{\mu}_{R}=6.9, \hat{\sigma}_{R}=2.6, n_{R}=44$ and $\hat{\mu}_{R_{H}}$ $=5.9, \hat{\sigma}_{R_{H}}=2.6, n_{R_{H}}=44$ and the MLEs of the normal prior is $\left(\hat{\theta}, \hat{\tau}^{2}\right)$ $=(6.4,0.0964)$. The mixture prior information can be adjusted by the weights of $\pi_{1}\left(\mu_{\mathrm{B}}\right)$ and $\pi_{2}\left(\mu_{\mathrm{B}}\right)$, respectively. However, the priors of the biosimilar and the innovator could be similar because of the similar manufacturing process of them. The selection of the weight in the mixture prior should be a small value, we choose $\gamma=(0,0.1,0.2)$. When $\gamma=0$, the mixture prior information is the prior information of the reference biological product. On the other hand, for the high similarity between the biosimilar and the innovator, the determination of limit, $\rho$ should be large enough to claim the biosimilarity. We suggest that the determination of limit, $\rho \geq 0.8$. In this study, we select $\rho=(0.8,0.9)$. Let $\Delta$ be the difference of treatment effect of the biosimilar and the prior mean of the innovator product, where $\Delta=\mu_{B}-\hat{\theta}$. We consider that $\Delta$ $=(-2,-1.5,-1,-0.5,0,0.5,1,1.5,2)$. For each combination of parameters $(\gamma, \rho, \Delta)$, the posterior probability $\mathrm{P}_{\mathrm{sp}}$ is calculated by Eqn. (5) through the numerical integration.

Table 1 exhibits the posterior probability $\mathrm{P}_{\mathrm{sp}}$ for the combinations of the parameters with $\hat{\sigma}_{R}=\hat{\sigma}_{B}=2.6$ and $\mathrm{n}_{\mathrm{B}}=44$. For instance, the first column in Table 1 corresponds to the posterior probability $\mathrm{P}_{\mathrm{sp}}$ with $\mu_{R}=6.9, \Delta=-2$ (i.e., $\hat{\mu}_{B}=4.9$ ), $\hat{\sigma}_{R}=\hat{\sigma}_{B}=2.6$ and $\mathrm{n}_{\mathrm{R}}=\mathrm{n}_{\mathrm{B}}=44$. Given $\rho=0.8$, the posterior probability $\mathrm{P}_{\text {sp }}$ for $\gamma$ equal to $0,0.1$, and 0.2 are, respectively, $0.9488,0.8539$, and 0.7590 . If we choose $\lambda=0.8$, we would claim biosimilarity on efficacy for the biosimilar when $\mathrm{Pr}$ $\left(\rho \mu_{R}<\mu_{B}<\mu_{R} / \rho\right) \geq 80 \%$. For example, when $\Delta=0.5, \rho=0.9$ and $\gamma=0.1$, the $\mathrm{P}_{\mathrm{sp}}$ is equal to 0.8257 and lager than 0.8 , the biosimilarity would be concluded.

As seen from Table 1, we observed that the $\mathrm{P}_{s p}$ decreases as the absolute value of $\Delta$ increases if $\rho$ and $\gamma$ are fixed. This indicates that the biosimilarity would be difficult to be claimed if the copy version is 
much different to the innovator product; otherwise, the biosimilarity would be easier to be claimed if the biosimilar is much similar to the innovator. The result also demonstrates that the $\mathrm{P}_{\mathrm{sp}}$ decreases as the determination of limit $\rho$ increases. This makes intuitive sense since the width of the similarity criterion $\rho \mu_{\mathrm{R}}<\mu_{\mathrm{B}}<\mu_{\mathrm{R}} / \rho$ is narrower as higher $\rho$ (more stringent). We also observed that the phenomenon that $\mathrm{P}_{\mathrm{sp}}$ is decreasing as the absolute value of $\Delta$ is increasing when $\rho=0.9$. The narrow width of $\rho \mu_{R}<\mu_{B}<\mu_{R} / \rho$ will be helpful to distinguish that the high biosimilarity between the biosimilar and the reference product is existing or not. This evidence supports that $\rho$ should be large to assess the high similarity between the biosimilar and the innovator.

\section{Real Example}

Human growth hormone (hGH) is produced by the anterior pituitary gland and is essential for normal growth in children. In United States, growth hormone deficiency (GHD) affects approximately one in 3,500 children. Recombinant human growth hormone (rhGH), an artificial form of hGH, is used as therapy for GHD and by body builders to improve muscle tone and size.

Genotropin is an rhGH approved in the EU in 1988 [16]. According to the paper of Wilton and Gunnarsson [17], the average height velocity, in 149 prepubertal children with GHD, increased from 3.3 to $9.3 \mathrm{~cm} / \mathrm{yr}$. Genotropin is widely used as a replacement therapy of hGH for children with GHD or Prader-Willi syndrome (PWS). Results are summarized in Table 2.

In the EU, two copy versions of Genoropin were approved to the market in 2006. One is Omnitrope, another is Valtropin. Romer et al. [18] conducted a clinical trial for comparing the efficacy and safety of Omnitrope with these of Genotropin. Omnitrope, which is approved by U.S. FDA, is a biosimilar product of Genotropin. In the trial of Romer et al. [18], 89 prepubertal children with GHD were participated and randomized to Genotropin group and Omnitrope group. The average height velocity increased from 3.8 to $10.7 \mathrm{~cm} / \mathrm{yr}$ in 44 children receiving Genotropin. In the other 45 children receiving Omnitrope,

\begin{tabular}{|c|c|c|c|c|c|c|c|c|c|}
\hline \multicolumn{10}{|c|}{$\rho=0.8$} \\
\hline \multicolumn{10}{|c|}{$\Delta=\mu \mathrm{B}-\hat{\theta}$} \\
\hline Y & -2 & -1.5 & -1 & -0.5 & 0 & 0.5 & 1 & 1.5 & 2 \\
\hline 0 & 0.9488 & 0.9878 & 0.9980 & 0.9998 & 1.0000 & 0.9999 & 0.9991 & 0.9956 & 0.9833 \\
\hline 0.1 & 0.8539 & 0.8890 & 0.8982 & 0.8998 & 0.9000 & 0.8999 & 0.8992 & 0.8961 & 0.8850 \\
\hline 0.2 & 0.7590 & 0.7903 & 0.7984 & 0.7998 & 0.8000 & 0.7999 & 0.7993 & 0.7965 & 0.7867 \\
\hline \multicolumn{10}{|c|}{$\rho=0.9$} \\
\hline \multicolumn{10}{|c|}{$\Delta=\mu \mathrm{B}-\hat{\theta}$} \\
\hline Y & -2 & -1.5 & -1 & -0.5 & 0 & 0.5 & 1 & 1.5 & 2 \\
\hline 0 & 0.3444 & 0.5745 & 0.7802 & 0.9075 & 0.9494 & 0.9174 & 0.8137 & 0.6424 & 0.4345 \\
\hline 0.1 & 0.3100 & 0.5171 & 0.7022 & 0.8168 & 0.8544 & 0.8257 & 0.7323 & 0.5781 & 0.3911 \\
\hline 0.2 & 0.2755 & 0.4596 & 0.6241 & 0.7260 & 0.7595 & 0.7339 & 0.6510 & 0.5139 & 0.3476 \\
\hline
\end{tabular}

Table 1: The results of posterior probability $P_{s p}$ for different combinations of $(\gamma, \rho$, $\Delta$ ) with $\hat{\sigma}_{B}=2.6, \mathrm{n}_{\mathrm{B}}=44$.

\begin{tabular}{|c|c|c|c|}
\hline \multirow{2}{*}{} & \multicolumn{2}{|c|}{ Romer et al. } & $\begin{array}{c}\text { Wilton and } \\
\text { Gunnarsson }\end{array}$ \\
\cline { 2 - 4 } & $\begin{array}{c}\text { Genotropin } \\
\mathrm{N}=45\end{array}$ & $\begin{array}{c}\text { Omnitrope } \\
\mathrm{N}=44\end{array}$ & $\begin{array}{c}\text { Genotrope } \\
\mathrm{N}=149\end{array}$ \\
\hline $\begin{array}{c}\mathrm{HV}, \begin{array}{c}\text { mean(SD), cm/ } \\
\text { year }\end{array} \\
\text { Baseline }\end{array}$ & $3.8(0.8)$ & $3.9(1.2)$ & $3.3(1.4)$ \\
\hline Study End & $10.7(2.9)$ & $10.7(2.6)$ & $9.3(2.6)$ \\
\hline
\end{tabular}

Table 2: Summary of results. the height velocity increased from 3.9 to $10.7 \mathrm{~cm} / \mathrm{yr}$. Details are listed in Table 2. We use the result in the paper of Wilton and Gunnarsson [17] and that in the paper of Romer et al. [18] to illustrate the empirical Bayes method for assessing biosimilarity.

The result from Wilton and Gunnarsson [17] is regarded as the historical information of the innovator drug, Gneotrope. Therefore, we knew that $\left(\hat{\mu}_{R}, \hat{\sigma}_{R}, n_{R}\right)=(6.8,3.01,45)$ and $\left(\hat{\mu}_{R_{H}}, \hat{\sigma}_{R_{H}}, n_{R_{H}}\right)=(6,2.95$, 149). The MLEs of the normal prior is $\left(\hat{\theta}, \hat{\tau}^{2}\right)=(6.2549,0.0346)$. We choose the weight in the mixture prior equal to 0.1 , i.e., $\gamma=0.1$. We have a mixture prior information of $\mu_{\mathrm{B}}$ for the biosimilar,

$$
\pi\left(\mu_{\mathrm{B}}\right)=0.1^{*} \mathrm{~N}(0,0.0346)+0.9^{*} \mathrm{~N}(6.2549,0.0346) .
$$

For assessing high similarity, the limit for allowing the similarity is selected as $\rho=0.9$. If the posterior probability $\mathrm{P}_{\mathrm{sp}}$ is larger than $80 \%$ (i.e., $\lambda=0.8$ ), we will conclude that Omnitrope and Gentropin are biosimilar. When $\gamma=0.1$ and $\rho=0.9$, the posterior probability $\mathrm{P}_{\mathrm{sp}}$ is 0.8952. Thus, we claim that Omnitrope is similar to Genotropin based on the proposed empirical Bayes method.

\section{Conclusion}

In this article, we establish a posterior criterion to assess the biosimilarity of two biological products using a mixture empirical prior information. Based on the proposed biosimilarity criterion, the biosimilarity will be concluded if the posterior probability is higher than a pre-specified level, say 80 or $90 \%$.

In our method, we need to select an appropriate value of $(\gamma, \rho, \lambda)$. We expect that the priors of the biosimilar and the innovator could be similar because of the similar manufacturing process of them. Thus, the weight of the mixture prior, $\gamma$, could be a small value but keeps the flexibility of prior determination. In this study, we set that $\gamma \leq 0.2$. Second, we need to choose the limit $(\rho)$ for the biosimilarity criterion to judge whether the biosimilar is similar to the innovator biologic. The determination of limits $\rho$ decides the accuracy of the biosimilarity and should be large enough to confirm the accuracy. The larger $\rho$ results in a more stringent biosimilarity criterion. Otherwise, the smaller $\rho$ leads to looser biosimilarity criterion. In this research, we set that $\rho \geq 0.8$. Finally, in order to claim high similarity between the biosimilar and the innovator, the pre-specified limit $\lambda$ is suggested to be equal to or higher than $80 \%$.

The selection of $(\gamma, \rho, \lambda)$ plays an important role in the study. With an appropriate choice of $\gamma, \rho$ and $\lambda$, our method can reach a conclusion that the biosimilar is highly similar to the reference product when the differences in observed efficacy responses between the two biological products are very small. However, selection of $\gamma, \rho$ and $\lambda$ may be rather crucial and critical. The sponsor should discuss the determination of $(\gamma$, $\rho, \lambda)$ with the regulatory agency for conducting a biosimilar clinical trial.

\section{Acknowledgement}

The authors thank Miss Ya-Ting Hsu and Miss Chiung-Wen Tsui of National Health Research Institutes for their assistant, which edit this manuscript. This work was supported by grants from the National Health Research Institutes, Zhunan Taiwan [PH-104-PP-11, PH-105-PP-11, PH-106-PP-02] and the Ministry of Science and Technology of Republic of China [MOST 104-2118-M-400-002].

\section{References}

1. Evaluate (2017) Pharma World Preview, Outlook to 2022.

2. Biosimilars Market by Product (Recombinant Non-glycosylated Proteins (Insulin, rHGH, Interferon), glycosylated (mAb, EPO), Peptides (Glucagon, Calcitonin), Manufacturing (In-House, CMO) \& Application (Oncology, Blood Disorders) - Global Forecast to 2021. 
Citation: Tsou HH, Chen CT, Hsiao CF, Cheng YC (2018) Evaluation of Biosimilarity Based on an Empirical Bayes Method. J Bioequiv Availab 10: 7-10. doi: 10.4172/jbb. 1000367

3. The European Medicines Agency Evaluation of Medicines for Human Use (2015) Guideline on similar biological medicinal products containing biotechnology-derived proteins as active substance: non-clinical and clinical issues. European Medicine Agency, UK.

4. Food and Drug Administration (2015) Scientific Considerations in Demonstrating Biosimilarity to a Reference Product: Guidance for Industry. U.S. Department of Health and Human Services Food and Drug Administration Center for Drug Evaluation and Research (CDER), Center for Biologics Evaluation and Research (CBER).

5. Chen CT, Tsou HH, Hsiao CF, Lai YH, Chang WJ, et al. (2017) A tolerance interval approach to assessing the biosimilarity of follow-on biologics. Stat Biopharm Res 9: 286-292.

6. Chow SC (2011) Quantitative evaluation of bioequivalence/biosimilarity. J Bioequiv and Bioavailab S1.

7. Chow SC, Liu JP (2010) Statistical assessment of biosimilar products. J Biopharma Stat 20: 10-30.

8. Chow SC, Yang LY, Starr A, Chiu ST (2013) Statistical methods for assessing interchangeability of biosimilars. Stat Med 32: 442-448.

9. Hsieh TC, Chow SC, Liu JP, Hsiao CF, Chi E (2010) Statistical test for evaluation of biosimilarity in variability of follow-on biologics. J Biopharm Stat 20: $75-89$
10. Hsieh TC, Chow SC, Yang LY, Chi E (2013) The evaluation of biosimilarity index based on reproducibility probability for assessing follow-on biologics. Stat Med 32: 406-414.

11. Liao JJZ, Darken PF (2013) Comparability of critical quality attributes for establishing biosimilarity. Stat Med 32: 462-469.

12. 12. Liao JJZ (2015) A constrained non-inferiority approach for assessing clinical efficacy to establish biosimilarity. Int J Clin Biostat Biom 1: 2-8.

13. Lin JR, Chow SC, Chang CH, Lin YC, Liu JP (2013) Application of the paralle line assay to assessment of biosimilar products based on binary endpoints. Stat Med 32: 449-461.

14. Tsou HH, Chang WJ, Hwang WS, Lai YH (2013) A consistency approach for evaluation of biosimilar products. J Biopharma Stat 23:1054-1066.

15. Hsiao CF, Hsu YY, Tsou HH, Liu JP (2007) Use of Prior Information for Bayesian Evaluation of Bridging Studies. J Biopharma Stat 17: 109-121.

16. An Z (2009) Therapeutic monoclonal antibodies: From bench to clinic. Hoboken N.J. John Wiley \& Sons.

17. Wilton $P$, Gunnarsson R (1988) Clinical experience with gsenotropin in growth hormone deficient children. Acta Paediatr Scand Suppl 343: 95-101.

18. Romer T, Peter F, Saenger P, Starzyk J, Koehler B, et al. (2007). Efficacy and safety of a new ready-to-use recombinant human growth hormone solution. $J$ Endocrinol Invest 30: 578-589. 